\title{
qp: A Tool for Generating 3D Models of Ancient Greek Pottery
}

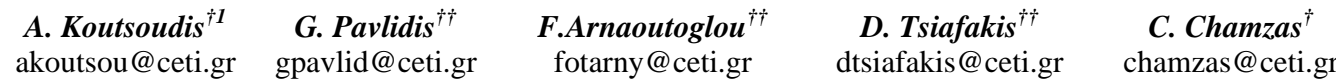 \\ † Democritus University of Thrace, Faculty of Electrical and Computer Engineering, Xanthi,67100, \\ Greece \\ ††Cultural and Educational Technology Institute/Research Centre 'ATHENA', Tsimiski 58, Xanthi \\ 67100, Greece
}

\begin{abstract}
The development of content based retrieval mechanisms is a very active research area. Present studies are mainly focused on automating the information extraction and indexing processes. Usually for the development and evaluation of such mechanisms there is always a need for a ground-truth database. In this paper we present a software tool named $q p$ that is able to semi-automatically produce a collection of random 3D vessels, with morphological characteristics similar to those found in ancient Greek pottery, a ceramic group exhibited worldwide with great impact to scholars as well as general public. A 3D vessel collection has been produced by $q p$ and can be used as a test bed dataset for the development of shape-based 3D descriptors applicable to pottery. Additionally, $q p$ can be considered as a 3D vessel modelling software tool which can be used by people not related to computer graphics technology and particularly to 3D modelling.
\end{abstract}

Keywords: Hermite Cubic Splines/2D curve approximation/ancient Greek pottery/ VRML/XML/MPEG-7/OpenGL/3D extrusion/3D revolve/point cloud triangulation

\section{Introduction}

Over the last years, multimedia databases with content derived from our cultural heritage thesaurus, have become extremely popular [1]-[4]. The increasing interest in preserving the past (historical and cultural), is reflected on museums and cultural organisations

\footnotetext{
${ }^{1}$ This paper is part of the 03ED375 research project, implemented within the framework of the "Reinforcement Programme of Human Research Manpower" (PENED) and co-financed by National and Community Funds (25\% from the Greek Ministry of Development-General Secretariat of Research and Technology and 75\% from E.U.-European Social Fund)
} 
which prioritise the production of digital archives of their collections. Pottery is the most representative category of objects related to cultural heritage and is found in most museums and archaeological sites. Several 3D digitised pottery artefacts are already available on the web. This is due to the fact that 3D scanning technologies continuously evolve and at the same time we progress into a mature era of computer graphics [5]. Different 3D scanning methodologies provide key answers to one of the primary challenges in computer graphics design; that is the generation of realistic detailed 3D replicas. Real world objects are usually approximated using triangulated meshes. This data structure is considered as the dominant format supported by all commercial 3D graphics hardware. It is a fact that the information provided by 3D models is superior to that of any other form of iconography, as it may lead to the physical replication of the object by means of 3D printing [6]. This has become clear also to non-experts who deal professionally with pottery, such as scholars in the field of culture and ceramists, and they would like to benefit of 3D models.

The content richness provided by 3D digitised artefacts has to be taken into account when using content based retrieval algorithms. Attempting to retrieve 3D models using textual annotation would not work properly in many cases. In fact, it requires much effort to identify and describe all possible aspects of interest in textual form manually and it is also rises the issue of which languages are going to be used. Current research in shape retrieval has produced mechanisms that guarantee better results when exploiting 3D shape properties [7][8]. One can understand, nowadays, that apart from dealing with 'How do we construct detailed $3 D$ replicas?' we need to address issues such as 'How can we retrieve them?' and more important 'How can we index them?'. The answers to such issues lie on:

- The development of algorithms that extract digital signatures from 3D models.

- Software that exploits those signatures and automatically or semi-automatically indexes and classifies them. 
- Sophisticated Graphical User Interfaces (GUIs) that allow different types of queries e.g. sketch-query, query-by-example.

In order to develop the aforementioned algorithms there is a need for a testbed; a groundtruth multimedia database, which allows the experimentation and performance evaluation of novel retrieval algorithms. To this end, we have implemented a software tool called $q p$. Its primary purpose is to semi-automatically produce a ground-truth dataset of 3D vessels in the common VRML 2.0 standard format. The produced 3D models exhibit morphological characteristics similar to those found in certain shape categories of ancient Greek vases. Moreover, they are accompanied by dynamically generated XML metadata (MPEG-7 compatible). Those metadata are referred to various characteristics related to the vessel but they also contain the MPEG-7 3D Shape Spectrum descriptor of each object. This will provide a comparison basis against other 3D object retrieval descriptors. On the other hand, the simplistic GUI of the produced software makes the tool useful to individuals that are not familiar with 3D modelling and would like a simple software tool to produce 3D pots by themselves.

The paper is organised as follows: In section 2, we outline the importance of pottery in the study of the ancient Greek culture with references to the morphological characteristics of vessels, the role of pottery in understanding ancient Greek culture, the impact of software tools in procedures followed by the cultural scientific community and how does $q p$ fits within this context. Then, we continue with a detailed description of the software application and its functionality, followed by section 4 where we discuss the procedure of randomly generating 3D vessels. In section 4, we present the 'Three Dimensional Vessel Description (ThreeDVDS)', a basic MPEG-7 compatible schema which describes a set of metadata. Finally, we conclude in section 5 by giving some thoughts on the future development of the software. 


\section{The Importance of Ancient Greek Pottery Study}

The ancient Greek vases, well known worldwide, have been studied and collected since the Renaissance and they are exhibited in museums internationally enjoying a great interest from both scholars and general public. Ancient Greek pottery had a long development and a remarkable continuity. Because of its hardy nature, its survival is abundant and it has exerted a large influence on understanding the society that produced the vases.

To understand the importance of clay and pottery in the lives of ancient Greeks, one need only to consider the many and various objects in use today that are equivalent to those earlier ceramic products, e.g. buckets, saucepans, light bulbs, clocks, jewel boxes, perfume sprays, wine bottles, tankards, toys, feeding bottles, roof tiles, beehives. Most of the Greek pottery was not shaped for display or as an art object but it was meant to serve one or more functions related to private and social life. Vases played a crucial role in matters of life and death, domestic tasks, rituals, leisure and pleasure as well as in political, social, and economic background in which they were produced.

One of the major aspects in studying an ancient Greek vase is its shape, that itself remains a valuable source of information regarding both private and public life. Their shapes are directly related to the functions that they were created to perform forming that way preserved records with data regarding ancient Greek life. The structure of a shape is generally likened to the human anatomy: mouth, neck, shoulder, body, and foot. Worth of note is that the Greeks called 'ears' the handles of the vase. Pots were not always made in one piece but were thrown in separate sections that were joined together with clay slip by the potter. This is also true for the way a pot is being modelled within $q p$ as two 
parametric curves known as splines are used to describe the main body and the handles of a vessel.

Worth of note is that as a general rule, potters adhered to traditional shapes, unless they were persuaded that a change was an advantage: easier to form, more saleable, answering new needs. The basic shapes of Greek pottery vary according to the geographical production area. It is through those variations that scholars can assign to different sources the material found and to observe the influence of one area upon another. Furthermore, pottery shapes varied through time. Their development was based on certain methodologies such as radically or in details and from time to time new shapes were introduced. Here again, the study of shape-changes enables a framework of relative chronology and influence to be erected.

Due to all the above, ancient Greek Pottery is considered as one of the major research fields in Archaeology. Numerous Greek vases are exhibited in museums or studied in detail by scholars of various fields. Archaeologists, classicists, art historians, students of classical literature, mythology, and history, social and economic historians and students of Greek religion are only some of the scholars that deal with ancient Greek vases. To them should be added a significant number of ceramists who reproduce ancient Greek vases. All of them consider the importance of the shape and make attempts to reconstruct a vessel, using so far drawings. It is well known that the study of pottery is based traditionally on drawings and photographs. This is due to the fact that a physical contact with the vase is usually limited and most important cannot be continuous. However, a drawing depicts only a profile and it cannot provide a real image of the vase. Photographs are also a major source for its study but they only depict certain viewpoints and again in 2D. 
The geometry of ancient Greek vases raised as an issue early in the archaeological research [22] and since Birkoff [41] studied geometry of vessels in 1933, many researchers have been focused on finding solutions that will put technology into service for archaeologists. Several projects have been motivated by the contemporary needs and requirements of archaeology in order to produce software applications that will provide methodology enhancements in identifying, documenting, archiving and visualising pottery data in both 2D and 3D. We find software tools which facilitate procedures such as:

i. Pottery fragment matching [33]

ii. Generic 3D vessel modelling [40]

iii. 3D vessel modelling based on profile images [31][32]

iv. Pottery curvature analysis, deformation measuring and classification [35][46]

v. 3D reconstructions based on three dimensionally scanned vessel fragments [32][34][37]-[39][43][44][47]

vi. Profile generation from fragments [45]

Those that are capable of reconstructing a vessel from its fragment usually require the use of a 3D scanning system in order to provide the reconstruction software with the appropriate datasets [32]-[35][37][39][42][43]-[46]. Others are based on both 3D scanning systems and commercial 3D modelling software [33]. Some have introduced the idea of free 3D modelling using Virtual Reality GUIs [40], while others rely their reconstruction on profile curve fitting [31]. $Q p$ can be found in the third category and although it doesn't improve the automation level of a ' $3 D$ reconstruction based on profile images' when compared with other published software tools such as [31] and [32], its main contribution is that it provides a mechanism for controlled randomisation and generation of random 3D vessels. The random generation procedure is considered as semi-automated due to the fact that the morphological characteristics of the generated 3D vessels are derived from parameters which are set by the user. It introduces the idea of exploiting the major morphological characteristic of vessels, which is the azimuthal 
symmetry along a specific axis, in order to generate multiple similar vessels accompanied by automatically generated metadata. Furthermore, the software uses Hermite B-Splines for the description of the profile which allows a low number of control points to be used for the accurate approximation of a profile.

\subsection{Shapes selected for qp}

Although not immense, the number of shapes found in ancient Greek pottery is fairly large [20]-[22]. In order to choose the shapes that will be used for the application, two major factors were initially taken under consideration: place and time. As it has been described earlier, these two factors influence the variation and development of the ancient Greek vases. Eligible for the project was a great range of shapes found commonly in ancient Greek pottery [20][21][29][30]. Using as key criteria the potentials offered until now by the software application and the aspiration to apply it to the most possible typical categories of pot shapes, the following shapes described in detail in Appendix I, have been chosen and presented in alphabetical order in table I:

\section{TABLE I}

Furthermore, the chosen shapes reflect a variation of aspects regarding ancient Greek culture. Daily life, male and female, cult and ritual, life and death, all major aspects of the ancient Greek culture, are represented through the selected vessels. Hence amphora, dinos/lebes, psykter, and phiale are related to one of the most characteristic activities of the ancient Greek life, the symposion (the male-only drinking party that followed dinner). Amphora was used to store the wine. Dinos/lebes was used for mixing wine and water, psykter was used for cooling wine while phiale was the drinking cup. Daily life and female activities are represented through kalathos, and plemochoelexaleiptron. Kalathos was a wool basket and as spinning was considered to be the chief occupation of women, kalathos is constantly mentioned in connection with them. In addition, kalathos was also 
used for cult purposes. As a perfume vase plemochoelexaleiptron was close related with another aspect of the female world. Alabastron was also a perfume vase used by women in domestic context as well as offering at tombs. Apart from the shapes themselves, there was a selection of certain types of each shape in order to present some of the most characteristic pottery workshops and techniques of the ancient Greek pottery. Archaic (7th-6th c. B.C.) and Classical (5th - 4th c. B.C.) periods provide the most renowned Greek vases found in museums all over the world. Athens and Corinth are two of the most important Greek pottery centres. Moreover, Athenian pottery has enjoyed a privileged position in the study of Greek pottery over the last century. During the Archaic times though, East Greek pottery -made along the coast of the Asia Minor and the nearby islands - enjoys a remarkable flourishing and expansion. All three of them, East Greek, Corinthian and Attic pottery, are represented through the selected types of each shape. A great chronological range from the 7th until the 5th c. B.C. is also covered through the chosen examples that represent both Archaic and Classical times.

\section{Modelling 3D Vessels with qp}

This section describes the architecture and the basic components of the developed application. Currently, the application is only available for the Microsoft Windows platform. The algorithms that have been developed are described in the following paragraphs.

\subsection{System design and operation analysis}

The four basic logical components of the application and their interconnections are depicted in figure 1. These are: 
a. The $3 D$ Component which contains the algorithms responsible for the 3D meshes generation.

b. The Vessel Profile Component that contains all the procedures related to the spline's mechanism.

c. The actual GUI component and

d. The File Handling and Exporting Component responsible for the generation of VRML 2.0 and XML files.

The connection lines (both solid and dotted) in Figure 1 illustrate the data flows between the logical components of the software application. The solid lines represent the logical intra-component relationships and the dotted lines the inter-components relationships.

Figure 1

$q p$ is designed to operate in two modes, either as a simple $3 D$ Vessel Lathe ( $3 D V L$ mode) or as a random $3 \mathrm{D}$ vessel generator ( $R 3 D M$ mode). In both cases, the generation of $3 \mathrm{D}$ vessels requires a small number of steps (procedures) to be followed. These steps ${ }^{2}$ include the following:

i. Initially a user can either :

a. Load a vessel's profile bitmap image which will be used as a reference.

b. Open a previously saved profile file for editing and further processing.

c. Design a new profile from scratch without any bitmap image reference.

ii. Considering the case of a new profile, the user manually approximates the vessel's profile (main body and handle) by deforming splines and by using the profile image as a guide. More specifically, the user defines initially a number of points that lie on the vessel's profile as it appears on the background bitmap (Figure 2). This is done simply by right clicking with mouse over the profile. At

\footnotetext{
${ }^{2}$ Steps (iv.) and (v.) are required in R3DM mode only.
} 
present, $q p$ allows the design of truthful and meticulous approximations but at present the system relies completely on the user's decision of whether the spline matches the profile. In cases where the profile includes a handle a second spline is used to match the handle. At present the software supports only vertical handles based on three types of intersection bases. Those are depicted in Figure 3. A vessel can have a maximum of two identical vertical handles. When the user is satisfied with the profile approximation, a complete 3D triangulated mesh of the vessel is automatically generated with a button click. The user can further modify the profile and observe the changes on the model's triangulated mesh in real time. The 3D model of the vessel can be examined by the user through the 3D viewer panel which is equipped with typical functionalities such as rotation on arbitrary axis and zooming in/out functions (Figure 4). The graphical user interface of $q p$ is depicted in Figure 4. It is organised into two main regions (Region A and B). Region A contains the profile editor panel and the 3D viewer panel. On the other hand, region B depicts the randomization control panel ( $R 3 D M$ mode) which is not required when using the software for modelling profile images ( $3 D V L$ mode). Thus, the minimal GUI complexity of region A in combination with the low learning overhead results a straightforward solution to individuals that are not familiar with 3D modelling and demand a fast approach on producing vessels in three dimensions. Furthermore, the user can select from a set of predefined profiles. Those profiles include vessel types such as alabastron, kalathos, lebes, phiale, exaleiptron, psykter and amphora (TABLE I). Once a profile file is loaded, it appears on the profile editing window of the application and the user can start altering the profile. As the 3D models can be exported into VRML 2.0 standard file format, they can be used in other software applications (Figure 5). The profile of a vessel is saved in a custom text based format which contains only the profile control points coordinates. Additionally, the system can render JPEG images of the 3D viewer 
panel so that thumbnail images of the $3 \mathrm{D}$ model can be made available to the user.

\section{Figure 2 \\ Figure 3 \\ Figure 4 \\ Figure 5}

iii. In $R 3 D M$ mode, random vessels can be generated from the deformation of the vessel's profile. The profile deformation is controlled/limited by a set of two dimensional regions that are defined by the user. The coordinates of the control points of the splines are randomised within those regions resulting new profiles. Those regions can be separately defined for each control point of the profile and stored in a look up table. The definition of a 2D randomisation region is done by moving a control point to a legitimate minimum (down left corner of the 2D region) and maximum (top right corner of the 2D region) position. By right clicking on these positions, their coordinates are pre-stored by the software and then the user can actually include them in the randomisation look-up table or redefine them. Depending on the randomisation mode ${ }^{3}$ the software consults this table to retrieve the randomisation region of each control point. The limits of those regions are made visible to the user on the profile editor panel during the region definition phase or during the random vessel generation phase.

iv. In $R 3 D M$ mode, the user can define additional parameters regarding: (1) the height and width variations of the randomly generated vessels, (2) several entries of the accompanying metadata, and (3) the total number of random vessels to be produced.

$\mathrm{v}$. In $R 3 D M$ mode, both the $3 \mathrm{D}$ models and the accompanying metadata are automatically exported.

\footnotetext{
${ }^{3}$ Described in detail in section 3.3
} 


\subsection{Three Dimensional Vessel Generation}

According to Staudek [10], a vessel can be projected to its planar outline. In this paper, we are discussing the inverse projection. More specifically, $q p$ generates several shape types of vessels due to their symmetric morphological nature. These can be described by revolving their profile curve in a certain distance around a vertical axis. The vessel's profile approximation relies on parametric curves, also known as splines [11]-[13]. A spline can be specified as a special function defined piecewise by polynomials. Hermite splines [11]-[13] are a widespread type of cubic splines used to smoothly interpolate between given points. Hermite splines work in any number of dimensions. It is a third degree spline curve where each polynomial is in Hermite form. A Hermite form consists of two control points and two control tangents for each polynomial. In Figure 6, $P 1, P 2$ are the starting and ending points of the curve, $T 1$ is the tangent vector (direction and speed of how the curve leaves the starting point) and $T 2$ is the tangent vector (direction and speed of how the curve reaches the ending point).

Figure 6

On each Hermite curve, given a starting point $p_{1}$ at $t=0$ followed by ending point $p_{2}$ at $t=1$ with starting tangent $m_{0}$ at $t=0$ and an ending tangent $m_{l}$ at $t=1$, the Hermite interpolant $p(t)$ can be defined as

$$
p(t)=\left(2 t^{3}-3 t^{2}+1\right) p_{0}+\left(t^{3}-2 t^{2}+t\right) m_{0}+\left(-2 t^{3}+3 t^{2}\right) p_{1}+\left(t^{3}-t^{2}\right) m_{1}
$$

where $t \in[0,1]$ [11]-[13]. The four Hermite's polynomial basis functions are depicted in Figure 7.

Figure 7

Notice that at $t=0$ only the $H_{0}(t)$ function affects the curve as all the other functions are zero. As soon as $t$ becomes greater than zero, $H_{1}(t), H_{2}(t), H_{3}(t)$ begin to have influence. In order to approximate a vessel's profile, sequential Hermite curves can be used. Each 
curved is defined as a subinterval. Between two sequential subintervals there is a common control point (the ending point of the previous curve and the starting point of the next curve), thus forming a chain of Hermite curves. Figure 8 depicts such a case. Control point $P_{1}$ is the ending point of the first subinterval and the starting point of the second subinterval. In such cases the interpolation is also performed on one subinterval at a time.

Figure 8

Initially, the tangent vectors are automatically computed for each subinterval. All control points and tangent vectors can be manually adjusted so that the polylines describe the vessel's profile accurately. In figure 2, the dotted line on the left figure represents the background profile image whereas on the right side the spline has matched the curvature of the profile by moving the tangent vectors.

When the user is satisfied with the profile's approximation, the program returns a set of 2D points which describe the vessel's profile. Let $P$ be a set of points in a 2D Cartesian coordinates system that are produced by interpolating a set of subinterval Hermite polynomials that represent the main body of the vessel's profile. Similarly, let $P$ ' be a second set of 2D points that represent the vessel's handle intersection derived by another set of Hermite polynomials. The two sets $P$ and $P^{\prime}$ describe the profile of a vessel by discriminating the main body of the vessel from the handles.

The next step involves the simplification of sets $P$ and $P^{\prime}$ in order to allow the generation of 3D point clouds with varying density. The simplification is largely based on the Douglas-Peuker [14] algorithm which is applied on both data sets in order to reduce the total number of points while keeping the curvature details intact. The algorithm tries to preserve directional trends in a line using a tolerance factor (threshold) which may be varied according to the amount of simplification required in each case. The algorithm works recursively in the following manner. A distance threshold is initially defined (in the case of the application a value is given by default). Consider the solid ' $s$ ' type stripe in Figure 9 as $P$ (In this example $P^{\prime}$ is considered to be $n u l l$ - The vessel has no handles). 
A point (a) is selected as the fixed point and a point (b) as the floating point. The algorithm traverses through all the points of set $P$ by seeking the point with the maximum perpendicular distance from the line segment $a b$. This is true for point (c) (Figure 9). If the size of the line segment $f_{c}$ is higher than the given threshold, then point (c) is considered as an important point and it is kept. The algorithm marks point (c) as the new floating point and subsequently seeks the next point that defines the maximum perpendicular distance from the new line segment ac. As the line segment ed characterizes the maximum perpendicular distance from line segment $a c$ it is once again compared with the threshold value. If it is greater; then point $d$ is kept and once again the new point becomes the new floating point. The algorithm stops when all the points of $P$ are traversed [14]. The same simplification algorithm is also applied on $P^{\prime}$. The result is two subsets $P_{s}$ and $P_{s}^{\prime}$ for which the following are always true $P_{s} \subset P, P_{s}^{\prime} \subset P^{\prime}$.

\section{Figure 9}

The simplified version of $P, P_{s}$, is used to generate the vessel's main body. In figure 7, instances of $P_{s}$ are anticlockwise rotated by an angle $\theta \in[0,2 \pi)$ around the $Y$ axis of a right-handed 3D Cartesian coordinate system. Hence, the vessel's body is composed by rotated instances of $P_{s}$ in a 3D Cartesian coordinate system. This is a 3D modelling technique known as revolve or lathe. It can be found as surfaces of revolution in surface geometry literature. As the surface is generated by rotating a two-dimensional point set about a coplanar axis, an azimuthal symmetry is always achieved. The rotation step of angle $\theta$ can be chosen accordingly, allowing the generation of varying density 3D point clouds.

Figure 10

Apart from the main body, a significant number of vessels' shapes have lifting handles such as the ones occurred on the selected amphora type. In $q p$, the handles of a vessel are 
modelled using the extrude along path technique which is common in several commercial 3D modelling applications. At present, the software supports only vertical handles that evolve on the $\mathrm{X}-\mathrm{Y}$ axis plane (Figure 11). As shown in Figure 11, the handles follow a path that is defined by $P_{s}^{\prime}$. For each point in $P_{s}^{\prime}$ the system plots instances of one of the predefined handle basis (Figure 3). The type of the vessel's handle intersection basis is selected by the user.

Initially, each intersection instance is plotted on the $Z-X$ axis plane (Figure 11) with its centre at the origin of the coordinate system. Each intersection instance has to be rotated by a specific angle $\varphi$ about the Z-axis in order to generate the tubular form of the handle. The angle $\varphi$ is calculated by the approximation of the first derivative at each point of $P_{s}^{\prime}$ on the $X$ - $Y$ plane. More specifically, let $P_{1}^{\prime}$ and $P_{2}^{\prime}$ be two sequential points within $P_{s}^{\prime}$ with $\left(x_{1}, y_{1}\right)$ and $\left(x_{2}, y_{2}\right)$ the corresponding coordinates the angle $\varphi$ is given by: $\varphi=\arctan \left(\frac{y_{2}-y_{1}}{x_{2}-x_{1}}\right)-\frac{\pi}{2}$

The intersection is initially located on the origin of the Z-X plane. Then, it is rotated about the Z-axis according to $\varphi$, and finally it is translated so that its centre lies on the first of the two sequential points of $P_{s}^{\prime}\left(P_{1}^{\prime}\right)$. This is recursively repeated for all points in $P_{s}^{\prime}$. Additionally, the handle can be further parameterised in order to produce handles of different thickness ${ }^{4}$. The thickness of the handle is also affected by a dynamically calculated scale factor which is based on an exponential function. This scale factor provides enhanced realism to the final 3D model by giving thicker parts on the handle's bases (the points where they intersect with the vessel's main body).

Figure 11

\footnotetext{
${ }^{4}$ In cases where equations are used the parameterisation is performed by changing the variables (e.g. radius) but in cases of predefined point sets this is done by scaling the point set initial coordinates.
} 
The point clouds (main body and handles) are organised in two separated sorted data structures. The sorting approach results:

- All instances of $P_{s}$ and $P_{s}^{\prime}$ are organised sequentially.

- All points within each instance are indexed sequentially and reflect the order of appearance on the profile starting from the lowest point (closest to the origin) towards the highest point.

It should be noted that the software automatically relocates the first point of $P_{s}$ to zero coordinates on X-axis (horizontal) to ensure a watertight base of the model. These data structures allowed the development of a custom triangulation algorithm that exploits the a priori knowledge of the point cloud in order to be more efficient. The algorithm recursively generates a list with triads of vertices that each one composes a triangle. Those triads are identified by using as initial parameters the total number of $P_{s}$ and $P_{s}^{\prime}$ instances and the total number of points within an instance. Figure 12, illustrates the operation of triangulation algorithm and the way the triangles are generated. Initially, the base of the vessel is triangulated and then the algorithm continues in generating triangle stripes between the instances of $P_{s}$. In the case of the $P_{s}^{\prime}$, the base triangulation part is not performed as the handles are not watertight. Furthermore, in cases where the vessel carries a second handle, this is instantly generated by mirroring the coordinates of the first handle against the $X$ axis. Hence, no repetition of the triangulation procedure is required as the same vertex grouping is applicable on the mirrored handle. Thus, its performance allows a near real-time rendering of the 3D model as the user interacts with the profile curve. 


\subsection{Generating Random 3D Vessels}

In this section, we discuss the use of $q p$ as a random vessel generator. Various 3D vessels arise by setting random coordinates to the control points of the splines which describe the vessel's profile. As the content of $P$ and $P^{\prime}$ is affected by the positions of the control points, any displacement to those, will produce a different 3D model. However, this displacement has to be restricted so that the resulted 3D models have both logical and meaningful substance. At present, there are four randomisation modes (Figure 13):

i. Point Controlled Randomization mode (PCR): The coordinates of any control point are randomized within given limits (bounding boxes) which are defined by the user. In this mode, it is possible to have one bounding box for each randomized point.

ii. Leader-Followers Randomization mode (LFR): In this mode a control point plays the role of the leader and it is randomized within the limits of a bounding box defined by the user. Additionally, a subset of the control points are characterised as followers. In each randomization phase, the system computes the spatial difference between the old and new (random) coordinates of the leader. Then, it applies this spatial difference to the coordinates of the followers. Thus, it allows the randomization of a profile with the advantage of maintaining unaffected specific parts of the vessel's profile. This mode may be useful in cases where there is a need to avoid deformations in specific parts of a profile. Such parts might be the rim, the neck or even the base of a vessel. Furthermore, by applying the same spatial difference to the coordinates of the handle's control points, it is ensured that the handle bases will always be attached on the vessel's main body during randomization. 
iii. Height-Width Randomization (HWR) mode: The final 3D model is randomly scaled according to maximum and minimum scaling limits defined on the Yaxis (height) and $\mathrm{X}$-axis (width). The scaling factor can be different for each of the two axes allowing non-uniform scaling.

iv. Noise Randomization (NR) mode: The vertices of the 3D model are randomly displaced by a random value (Gaussian noise). This is considered as an approximation of the noise that exists on three-dimensionally scanned artefacts.

Figure 13

\section{3D Vessel Metadata - ThreeDVDS Schema}

We present an MPEG-7 compatible schema that can be adopted or extended by any MPEG-7 compatible database which contains 3D vessels. The schema is applicable to both computer generated and 3D digitised vessels. It is a basic set of metadata that accompany each 3D model that is produced with $q p$. The proposed schema can also be used for digital copies of real artefacts without the need of any modifications. When $q p$ is randomly generating vessels, all the elements described in the following schema (

TABLE II II) are automatically produced except from the comments entry which is left null unless the user provides some textual information manually. TABLE III lists the description of the metadata elements.

\section{TABLE II II}

\section{TABLE III}

The last metadata element contains the MPEG-7 3D Shape Descriptor. The MPEG-7 3D Shape Descriptor is based on the shape spectrum concept. The shape spectrum is defined as the histogram of the shape index, computed over the entire 3D surface. The shape index contains nine different shape categories where different areas of the 3D model surface are matched [15]. 


\section{System Performance Evalution}

The application performance was evaluated on an IBM compatible personal computer with an AMD Athlon 64 X2 4200+ processor and 2GB of RAM. The evaluation test of the revolve algorithm involved the generation of an amphora type vessel with splines of varying number of subintervals. This is achieved by increasing the number of control points (both control and tangent points) which affect the computational complexity of the algorithm. Figure 14 depicts the complexity of the spline, in the form of total number of control points, against the total number of vertices and the processing time required for the generation of the vessel's main body point cloud. An almost linear behaviour of the algorithm is observed.

\section{Figure 14}

The extrude along path algorithm was evaluated in a similar way by generating a concave type (Figure 3c) handle. The intersection basis of the handle was composed by 44 vertices. Once again, an almost linear behaviour of the algorithm is illustrated in Figure 15.

\section{Figure 15}

On the other hand, the behaviour of the triangulation algorithm is far from being linear and its performance is closer to an $O\left(n^{2}\right)$ class algorithm. Figure 16 depicts the total number of triangles in a 3D vessel against the processing time required for the triangulation.

Figure 16

We have used $q p$ to generate sets of random vessels based on different vessel shapes. For each shape a total of 50 models were generated. TABLE IV illustrates the performance of the system. The generation times include and the time required for saving the model in VRML 2.0 format. 


\section{TABLE IV}

\section{Conclusions}

In this paper, we discussed a software application, which is able to generate a groundtruth database of complete 3D vessels with emphasis placed on Greek Ancient Pottery. Of all kinds of artefacts which may play a significant role in human history and life, ceramics are surely of the most important. Clay vessels used for storing, cooking, serving food and carrying water, were first manufactured at least 12000-13000 years ago (perhaps a little earlier yet), and they continue to be manufactured for similar purposes until nowadays. We have tried to emphasise on the idea of 3D vessel parameterisation. An application was developed to provide rapidly a calibrated data set in terms of content categorisation; common coordinate systems and data structures that will be used for the development of shape based 3D descriptors focused on pottery artefacts that will provide means for automatic classification. A subset of basic 3D modelling approaches that can be found in many commercial 3D modelling software packages were identified and reengineered in combination with newly developed custom triangulation schemes and MPEG-7 functionality to be practically exploited in the blended research fields of information technology and cultural heritage. We have demonstrated the functionality of $q p$ by producing a number of random artificial 3D vessels based on some ancient Greek pottery shapes.

Additionally, $q p$ will be further extended in terms of modelling functionality and in terms of features such as automated profile extraction from 2D images; freeform handle intersection 2D editor, vessel volume calculation and texture mapping with the use of predefined texture maps tiles. We are also considering the use of Boolean operations and 3D skewing in order to generate incomplete vessels with eventual defects in workmanship. Additional functionality will allow the users to exchange a randomisation 
project file that will allow the re-generation of a given dataset and relieve the user from the need to download the whole dataset.

Concluding, $q p$ and the generated dataset can be found at http://www.ceti.gr/ akoutsou/qp. Furthermore, in order to simulate incomplete pots with eventual defects and errors that are usually found in 3D scanned models (laser stripe noise, occluded areas, etc) we batched processed the produced models with a commercial 3D scanner data processing software (Geomagic Studio 9). Thus, a second dataset, based on the first one, was generated where the models carry defects such as geometry discontinuities. These can also be found in the same website.

\section{References}

[1] E. Politou, I. Tsevremes, A. Tsompanopoulos, G. Pavlidis, A. Kazakis, C. Chamzas, Ark of Refugee Heirloom - A Cultural Heritage Database, Proceedings of Electronic Imaging \& Visual Arts EVA 2002 Conference, Florence, Italy, March 25-29, 2002.

[2] N. Tsirliganis, G. Pavlidis, A. Koutsoudis, D. Papadopoulou, A. Tsompanopoulos, K. Stavroglou, E. Politou, C. Chamzas, Integrated Documentation of Cultural Heritage through 3D imaging and multimedia database, Proceedings of International Symposium on Virtual Reality, in Proc. Archaeology and Cultural Heritage VAST 2001, Glyfada, Greece, Nov. 28-30, 2001.

[3] N. Tsirliganis, G. Pavlidis, A. Koutsoudis, E. Politou, A. Tsompanopoulos, K. Stavroglou, C. Chamzas, New Ways in Digitization and Visualization of Cultural Objects, Proceedings of IEEE International Digital Signal Processing Conference DSP 2002, Santorini, Greece, July 1-3, 2002, Vol. 1, pp. 475-478.

[4] G. Pavlidis, D. Tsiafakis, A. Koutsoudis, F. Arnaoutoglou, V. Tsioukas, C. Chamzas, Recording Cultural Heritage, Proceedings of Third International Conference of Museology, Mytilene, Greece, June 5-9, 2006. 
[5] H. Shiaw, R. J.K Jacob, Gregory R. Crane, The 3D Vase Museum: A new approach to context in a digital library, Proceedings of ACM/IEEE Conference on Digital Libraries (JCDL ’04), Tucson, Arisona, USA, June 7-11-2004, pp.125-134.

[6] M. Andreetto, N. Brusco, and G. M. Cortelazzo, Automatic 3D Modelling of Textured Cultural Heritage Objects, IEEE Transaction on Image Processing, vol. 13, no. 3, pp. 354-369, March 2004.

[7] Johan W. H. Tangelder, Remco C. Veltkamp. A Survey of Content Based 3D Shape Retrieval Methods, Proceedings of Shape Modelling International, 7-9 June, 2004, Genova, Italy. IEEE Computer Society.

[8] Faloutsos C., Searching Multimedia Databases by Content, Kluwer Academic Inc., 1996

[9] Karasik A, Mara H, Sablatnig R, Sharon I, Smilansky U., Measuring Deformations of Wheel-Produced Ceramics Using High Resolution 3D Reconstructions, Proceedings of the Computer Applications and Quantitative Methods in Archaeology Conference (CAA'05), 2005.

[10] Staudek, T., 'On Birkhoff's Aesthetic Measure of Vases. Technical Report', Faculty of Informatics, Masaryk University, Czech, 1999.

[11] I. Foley, van Dam, Feiner, Hughes, Computer Graphics - Principles and practice, Second Edition in C, ISBN 0-201-84840-6, pp.483-488

[12] Anne Morgan Spalter, The Computer in the Visual Arts, Addison Wesley Longman, ISBN 0-201-38600-3,1999,pp. 128-132

[13] Tomas Akeni-Moller, Eric Haines, Real-Time Rendering Second Edition, A.K. Peters LTD, ISBN 1-56881-182-9, 2002, pp. 492-494

[14] Douglas, D. H. \& Peucker, T. K., Algorithms for the reduction of the number of points required to represent a digitized line or its caricature. The Canadian Cartographer, v.10, 1973, pp. 112-122. 
[15] T. Zaharia and F. Preteux, 3D shape-based retrieval within the MPEG-7 framework, Proceedings of SPIE Conference on Visualization and Data Analysis, January 20-25, 2002, San Jose Convention Centre, San Jose, California, USA, pp 133-145.

[16] Sparkes, B. A., Greek Pottery: an introduction, Manchester 1991

[17] Sparkes, B. A., The Red and the Black: studies in Greek pottery, Routledge 1996

[18] Cook, R. M., Greek Painted Pottery, $3^{\text {rd }}$ ed. New York 1997

[19] Tiverios, M., Greek Art, Ancient Vases, (in Greek), Athens 1996

[20] Richter G.M.A. and Milne M.J., Shapes and Names of Athenian Vases, New York 1935

[21] Kanowski M.G., Containers of Classical Greece. A Handbook of Shapes, Queensland 1984

[22] Caskey, L. D., Geometry of Greek Vases, Boston 1922

[23] Angermeier, H. E., Das Alabastron: Beitrag zur Lekythen-Forchung, Giessen 1936

[24] Mauermayer, C. Das griechische Alabastron: Formgeschichte und Werwendung, München 1985.

[25] Brownlee, A., Attic Black-Figured Dinoi, Harvard 1981

[26] Luschey, H.., Die Phiale, München 1939

[27] Scheibler, I., Exaleiptra, $J d I$ (1964), 72-108

[28] Drougou, S., Der attische Psykter, Würzburg 1975

[29] Sparkes B.A. \& Talcott L., Black and Plan Pottery of the Sixth, Fifth and Fourth Centuries B.C. ', The Athenian Agora XII, Princeton 1970

[30] Moore M.B. \& Philippides M.Z.P., Attic Black-Figured Pottery, The Athenian Agora XXIII, Princeton 1986

[31] S. B Sanjay Goel, Siddharth Batra, Computer Vision Aided Pottery Classification and Reconstruction, INDO US Science and Technology Forum on Digital Archaeology, November, 2005 
[32] M. Kampel, R. Sablatnig, Virtual Reconstruction of broken and unbroken Pottery, Proceedings of the Fourth International Conference on 3 - D Digital Imaging and Modelling (3DIM’03), pp 2 - 6, January 2003

[33] I. Marie, H. Qasrawi, Virtual assembly of pottery fragments using moire surface profile measurements, Journal of Archaeological Science, Volume 32, Issue 10, pp 15271533, 1 October 2005

[34] M. Kampel, R. Sablatnig, On 3D Mosaicing of Rotationally Symmetric Ceramic Fragments, Proceedings of the 17th International Conference on Pattern Recognition, Vol.2, 2004, pp.265-268

[35] C. Maiza, V. Gaildrat, SemanticArchaeo: A Symbolic Approach of Pottery Classification, Proceedings of the International Conference Virtual Reality, Archaeology and Cultural Heritage (VAST 2006), Nicosia, Cyprus, 30/10/2006-04/11/2006, EPOCH Publication, October 2006, pp. 227-233

[36] J. Rowe, A Model Digital Library for 3D Pottery Data, ASU 3DK Native American Ceramic Vessel Digital Library Model, CNI Spring 2001 Task Force Meeting, Hilton Washington \& Tower, April 9-10, 2001

[37] F. Melero, A. León, F. Contreras, J.C. Torres, A new system for interactive vessel reconstruction and drawing , Bar International Series, 2004, VOL 1227, pp. 78-81 [38] C. Laugerotte, N. Warzée, An environment for the analysis and reconstruction of archaeological objects, 5th International Symposium on Virtual Reality, Archaeology and Cultural Heritage Eurographics Symposium Proceedings (VAST 2004), pp.175-183

[39] Sablatnig R., Menard C.,, "3D Reconstruction of Archaeological Pottery Using Profile Primitives", in: Sarris N., Strintzis M.G., "Proc. of International Workshop on Synthetic-Natural Hybrid Coding and Three-Dimensional Imaging",1997, pp. 93-96

[40] G. Han, J. Hwang, S. Choi, G. J. Kim, AR Pottery: Experiencing Pottery Making in the Augmented Space, HCI (14) 2007, pp. 642-650 
[41] G. D. Birkhoff, Aesthetic Measure, Cambridge Massachusetts' University Press, 1933

[42] H. Mara, M. Kampel, F. Niccolucci, R. Sablatnig, Ancient Coins \& Ceramics 3D and 2D Documentation for Preservation and Retrieval of Lost Heritage, Proceedings of the 2nd ISPRS International Workshop 3D-ARCH 2007: "3D Virtual Reconstruction and Visualization of Complex Architectures", ETH Zurich, Switzerland, 12-13 July 2007 [43] H. Mara, M. Kampel, Automated extraction of profiles from 3d-models of archaeological fragments, Proceedings of the CIPA 2003 XIXth International Symposium, 30 September - 04 October, 2003, Antalya, Turkey

[44] C. Maiza, V. Gaildrat, Automatic Classification of Archaeological Potsherds, Proceedings of the 8th International Conference on Computer Graphics and Artificial Intelligence, 3IA'2005, Limoges, France, 11 mai 12 mai 2005. Pléménos Dimitri (Eds.), Dimitri Pléménos, MSI Laboratory, Faculté des sciences, 83 rue d'Isle, 87000 LIMOGES, FRANCE, p. 135-147.

[45] A. Karasik, U. Smilansky, 3D Scanning Technology as a Standard Archaeological Tool for Pottery Analysis: Practice and Theory, Isaac Newton Institute Preprint Series, Analysis on Graphs and its Applications, 8 January - 29 June 2007

[46] H. Mara, R. Sablatnig, A. Karasik, U. Smilansky, The Uniformity of Wheel Produced Pottery Deduced from 3D Image Processing and Scanning, in: Burger W., Scharinger J., (Eds.), "Digital Imaging in Media and Education, Proceedings of the 28th Workshop of the Austrian Association for Pattern Recognition (OAGM/AAPR)", Schriftenreihe der OCG, 2004, Vol. 179, pp.197-204

[47] F.J. Melero, J.C. Torres, A. León, On the Interactive 3D Reconstruction of Iberian Vessels, Proceedings of the 4th International Symposium on Virtual Reality, Archaeology and Intelligent Cultural Heritage, VAST (2003)

[48] Empereur, J.-Y., Garlan, Y., Recherches sur les amphores grecques. Actes du colloque international (Athènes 10-12 Septembre 1984) / BCH Supp 13 (Athens 1986). 
[49] Timothy Darvill, Amphora, The Concise Oxford Dictionary of Archaeology. Oxford University Press, 2002.

\section{Appendix I: The chosen ancient Greek shapes (TABLE I)}

Alabastron: perfume-vase normally small in size that appears in various Greek workshops (e.g. Corinthian, East Greek, Attic) during the Archaic and Classical times ( $7^{\text {th }}-4^{\text {th }}$ c. B.C.). Alabastron has a broad, flat, disk-like mouth, a thin neck, a longish body shaped like a drop or elongated pear and it is usually footless. The vessel was used for women's toilet and for cult. The type used for the $q p$ application is the East Greek type which has a slim cigar-shaped body, it is pointed and handless and it dates during the $7^{\text {th }}$ and $6^{\text {th }}$ centuries B.C.

Amphora: The amphora is a type of two-handled ceramic vessel with narrow neck used for storage or transport. The shape is common in both plain and decorated wares. The Greek name amphoreus is an abbreviation of amphiphoreus (amphi= "on both sides", phoreus= "carrier"), referring to the two carrying handles of the vessel. The shape is divided into two major classes, neck-amphorae and one-piece amphorae. In neckamphorae neck meets body at an angle while in one-piece amphorae neck merges into shoulder and body in a curve. The type chosen for qp is a particular type of Athenian neck-amphora, the Panathenaic amphora that was designed to be filled with olive oil as a prize for the Panathenaic Games. Panathenaic amphorae were in use from about 560 B.C. to at least the second century B.C.

Plemochoe/exaleiptron: very distinctive shape: an oblate spheroid bowl with turned-in rim, short or tall foot, and lid with finial. It was a container perhaps for scented oil and the turned-in rim seems intended to prevent the contents from spilling out. It was a female vase as it is indicated also from the vase-paintings. The clay examples occur from the late sixth century B.C. and last until the second half of the fifth century B.C. The type used for $q p$ application is found in Attic pottery of about 500 B.C. 
Kalathos: a basket narrow at the rising to a larger size at the top. It is usually the basket in which women placed their work, and especially the materials for spinning (wool, spun and unspun). The type used for the $q p$ application is the Attic found in plain as well as in black figured technique and it dates in the 6th c. B.C.

Lebes/dinos: large, round-bottomed bowl without handles. It was used for mixing wine and water. The type used for the $q p$ application is found in Attic red figured pottery of the $5^{\text {th }}$ c. B.C.

Phiale: shallow bowl for wine that resembles a plate and it does not have either handles nor foot. The most typical form of phiale has a hollow knob in the middle (an omphalos, navel) projecting upward into the bowl. Phiale was used at the symposium as a drinking cup, while in ceremonies or religious rites was used to pour libations to the gods. The type was made at Corinth from about 600 B.C. on, and at Athens soon afterwards.

Psykter: mushroom-shaped vase either lidless and without handles or with a lid fitted to a flanged mouth and small tubes on the shoulder to accommodate strings for carrying the vase or, possibly, tying on the lid. It was used as a wine cooler. The shape was made at Athens from the last quarter of the $6^{\text {th }}$ c. B.C. or perhaps a little earlier, to the middle of the $5^{\text {th }}$ c. B.C. 


\section{Figures}

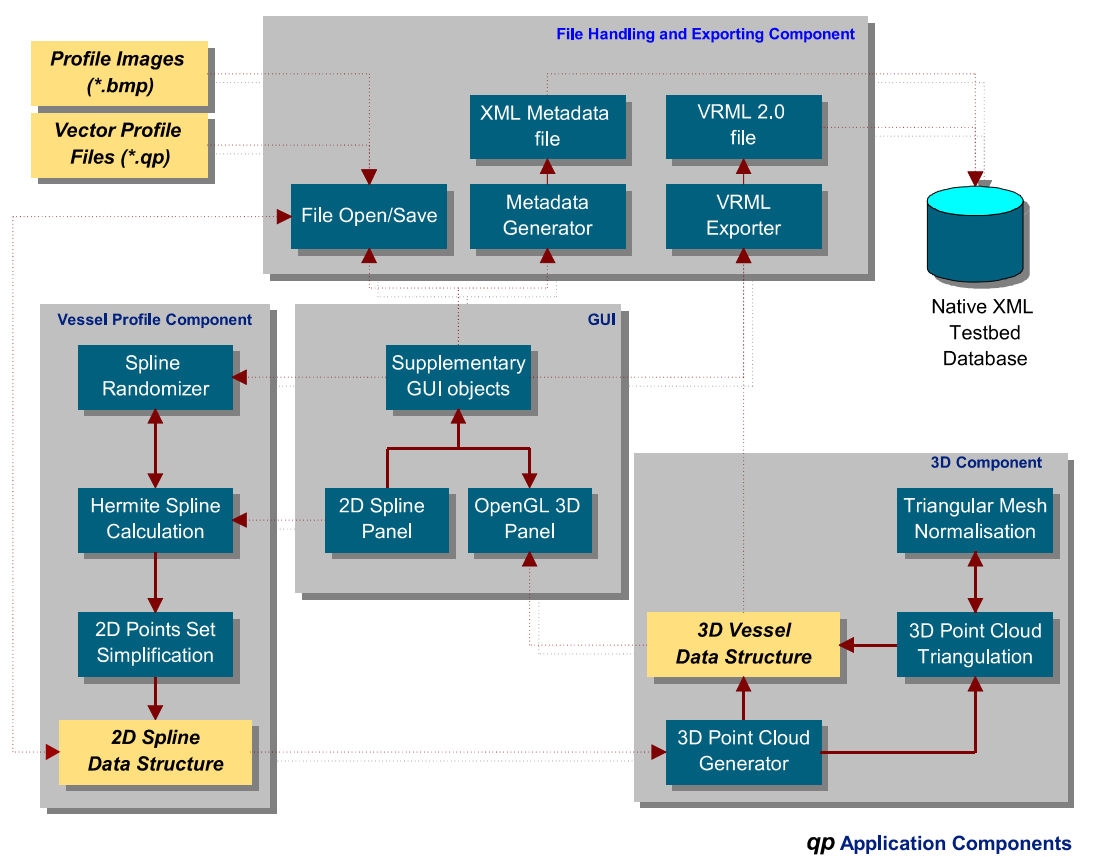

Figure 1 - Application architecture and data flow diagram
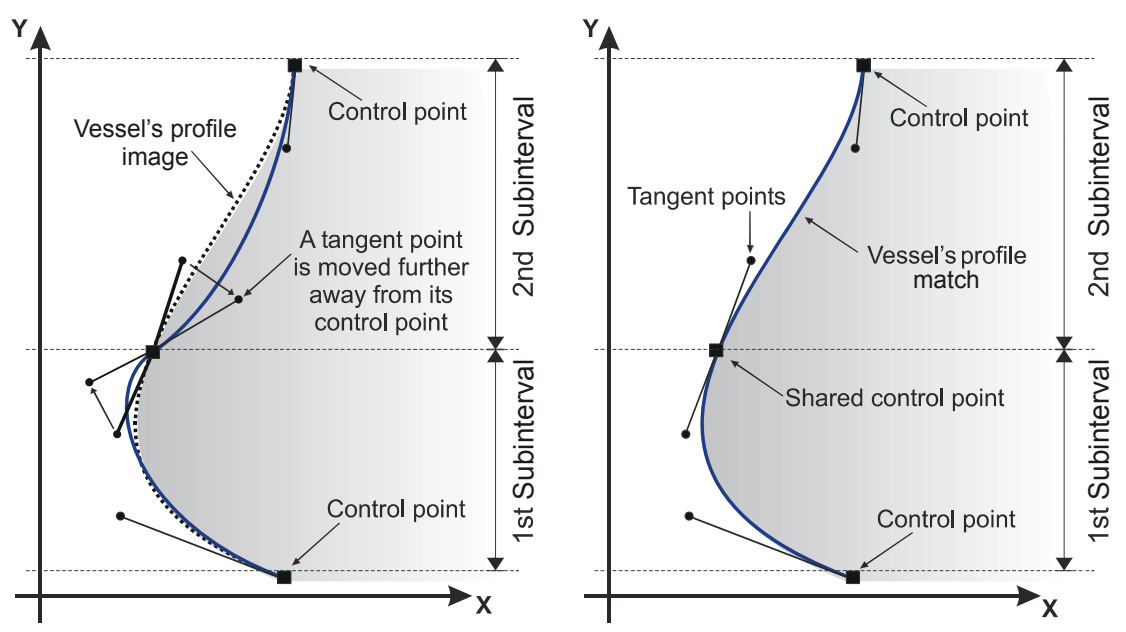

Figure 2 - Adjusting control points to deform the Hermite curves in order to approach vessel's profile. 


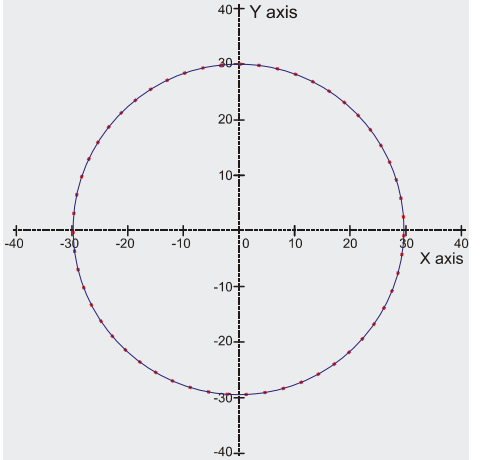

A. Basis for cylindrical type handle - Equation of circle

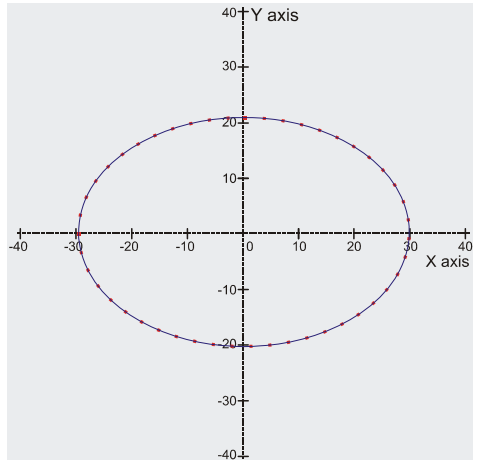

B. Basis for oval type handle - Equation of ellipse

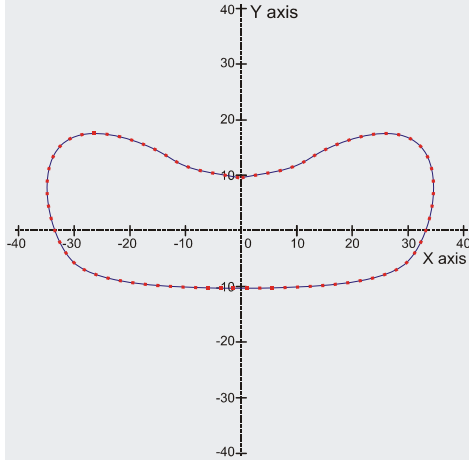

C. Basis for concave type handle - Predefined point

Figure 3 -Different handle types and their intersection basis. The user can parameterize the handle through the applications GUI

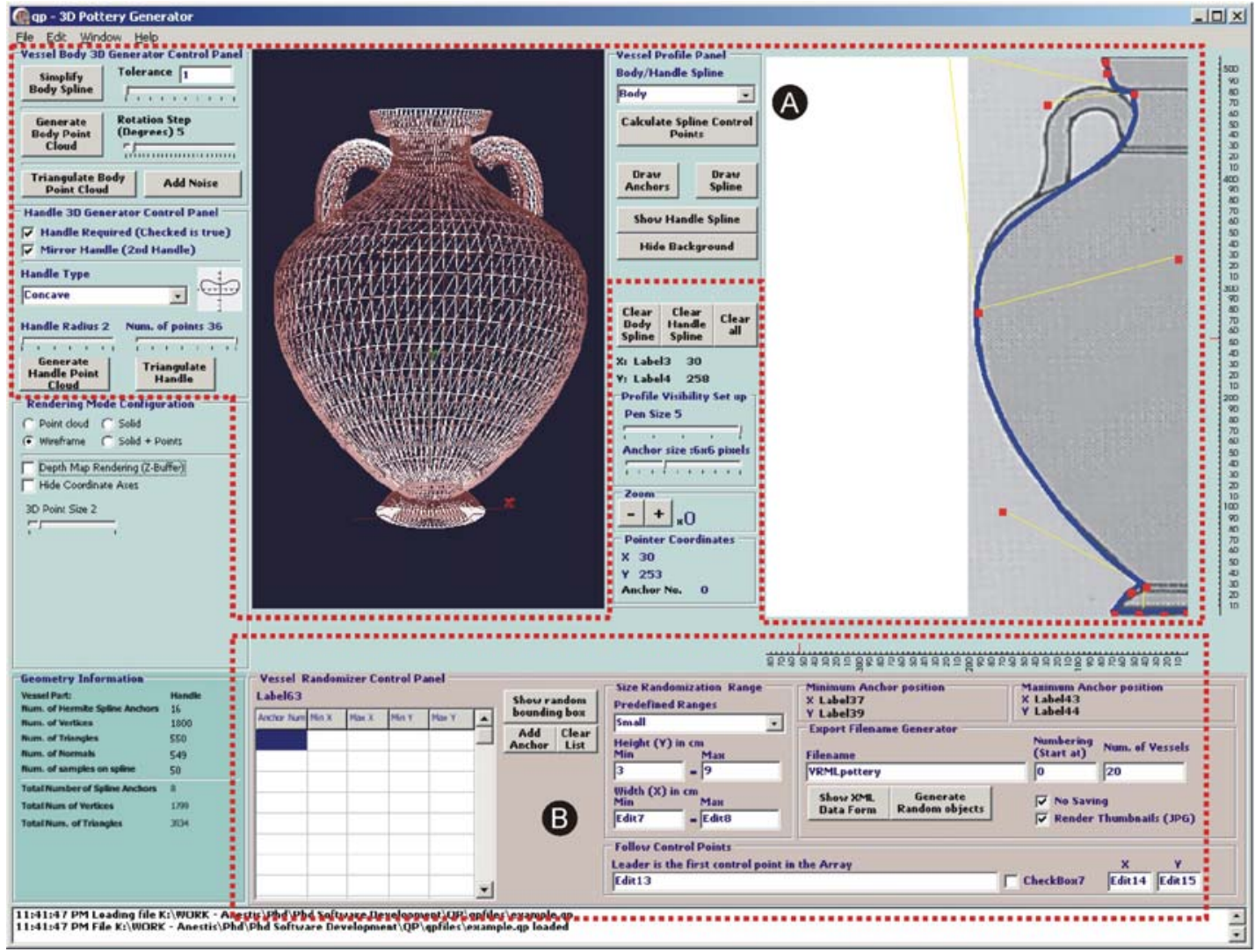

Figure 4 - The GUI of qp divided into two main regions. In region 'A', a $3 D$ vessel in wireframe rendering mode is depicted 


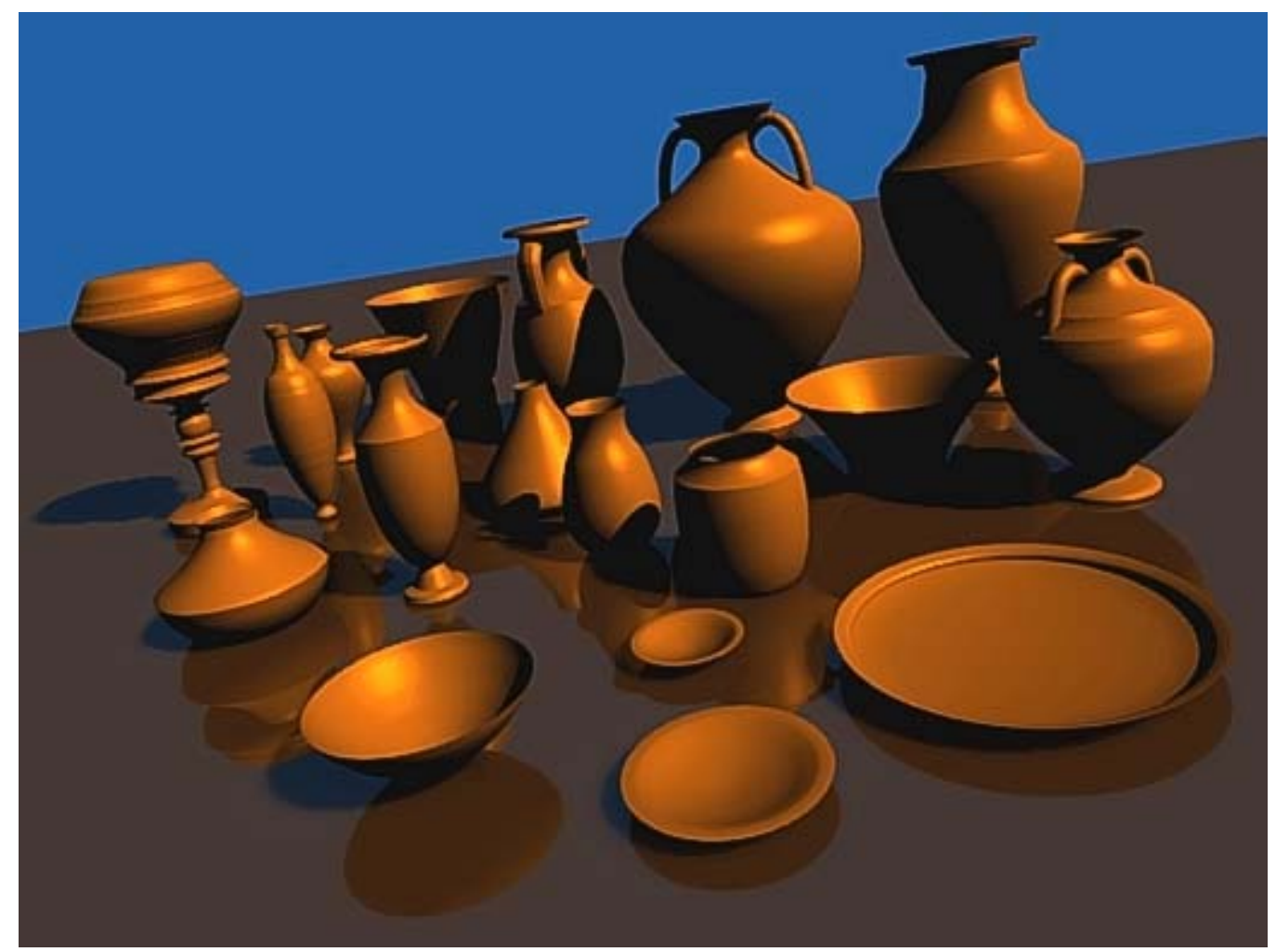

Figure 5 - Various $3 D$ Vessels generated by qp and rendered with Blender ${ }^{5}$

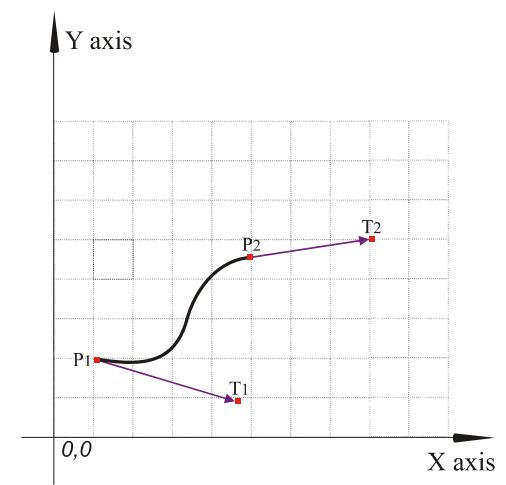

Figure 6 - A Hermite curve

\footnotetext{
${ }^{5}$ Blender is an open source 3D modeling software - http://www.blender.org
} 


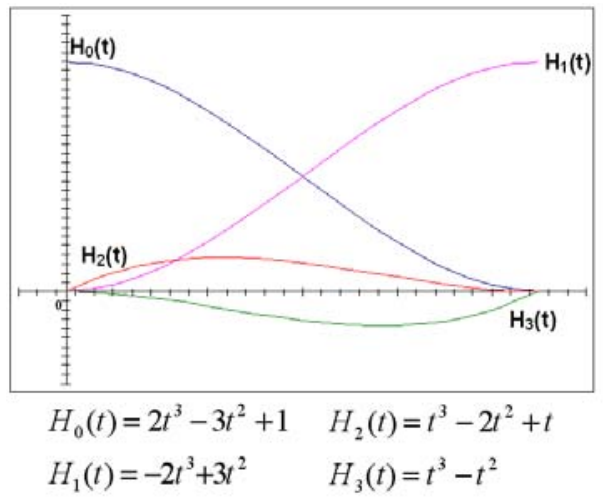

Figure 7 - The four Hermite basis blending functions

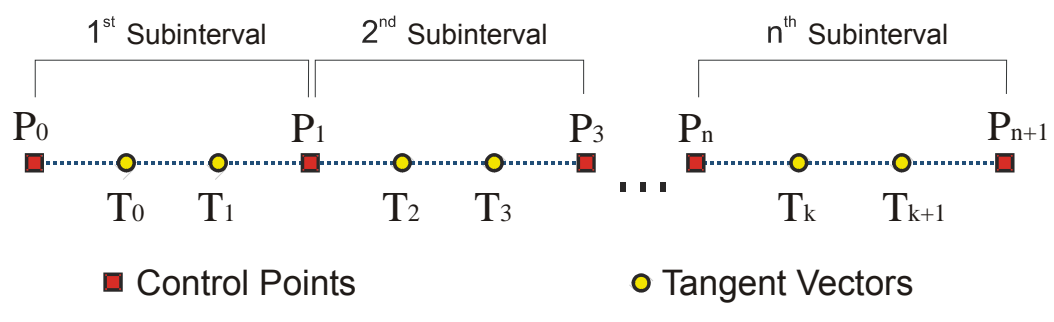

Figure 8 - A chain of $n$ Hermite Curves (subintervals)

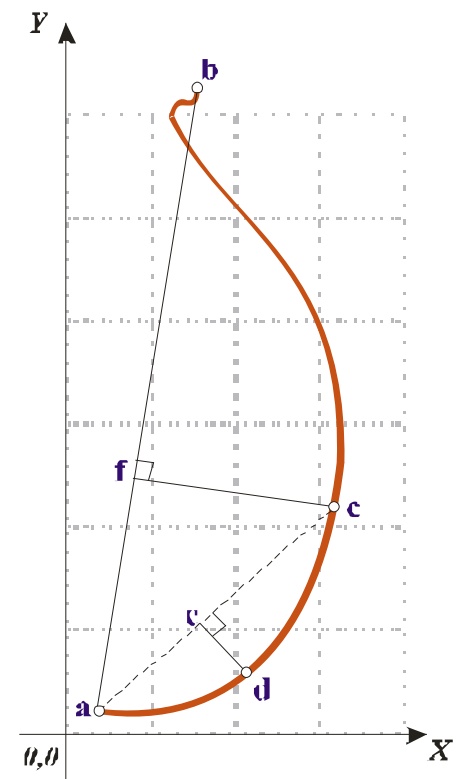

Figure 9 - Douglas - Peuker 2D simplification algorithm 


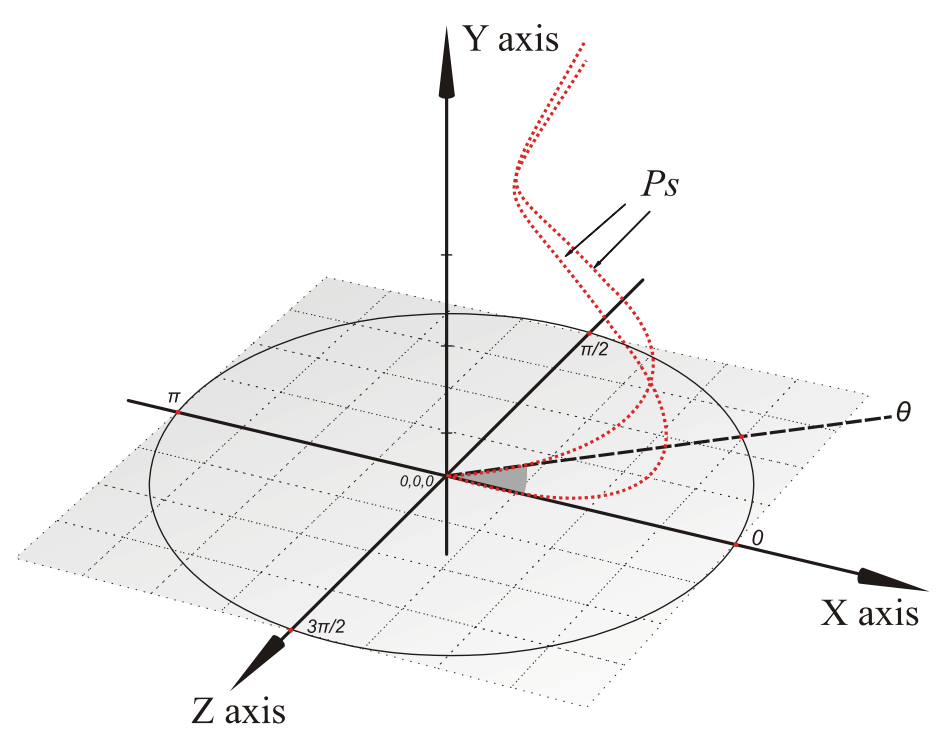

Figure 10 - Generation of Vessel's main body using the Revolve technique
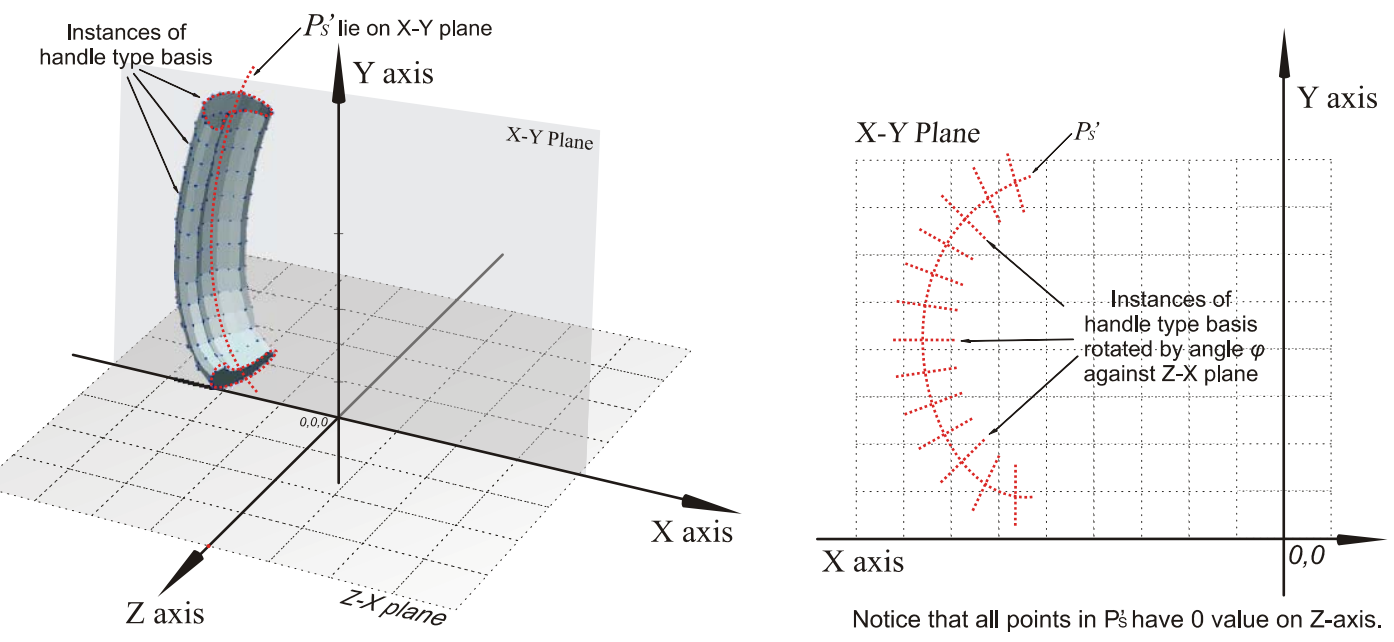

Figure 11 - Extrude along Path technique is used for the generation of a concave type handle
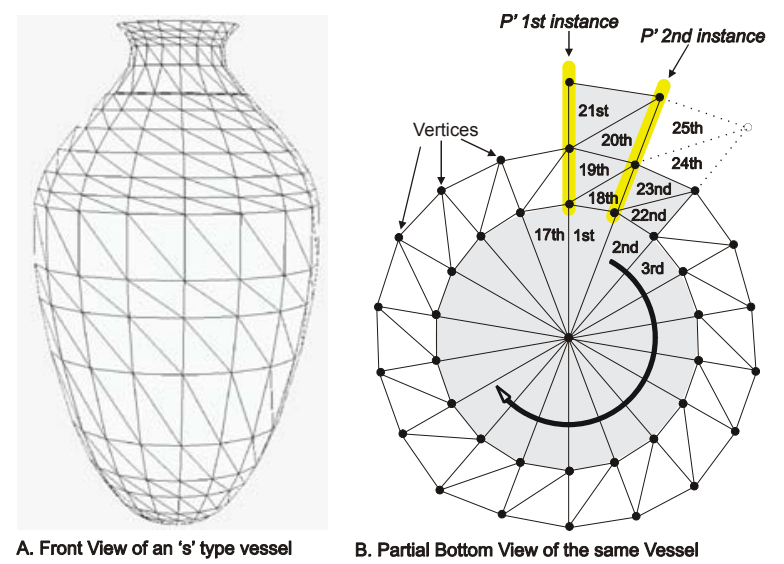

Figure 12 - Triangulation algorithm 
A. PCR Mode

$\mathrm{Y}$ axis

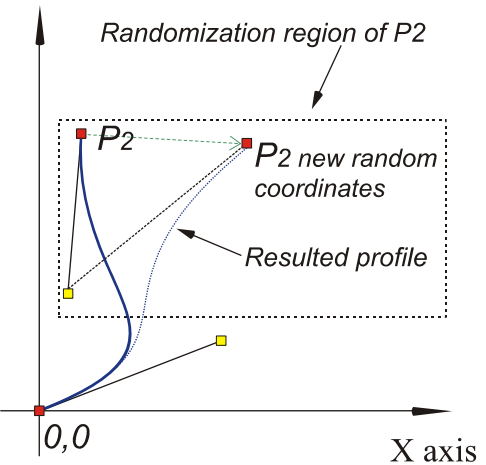

C. HWR mode

$\mathrm{Y}$ axis

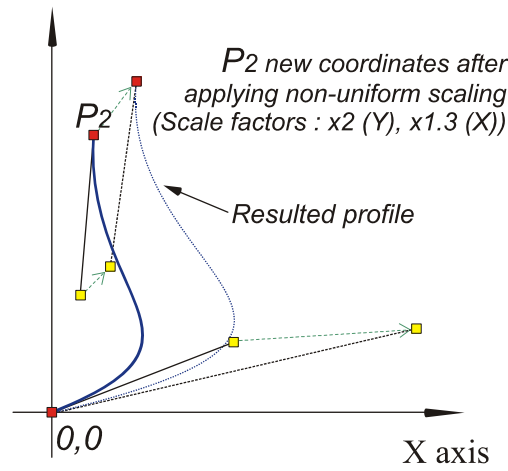

\section{B. LFR mode}

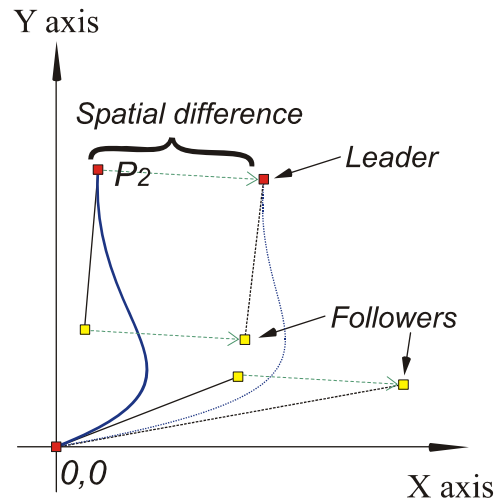

C. NR mode

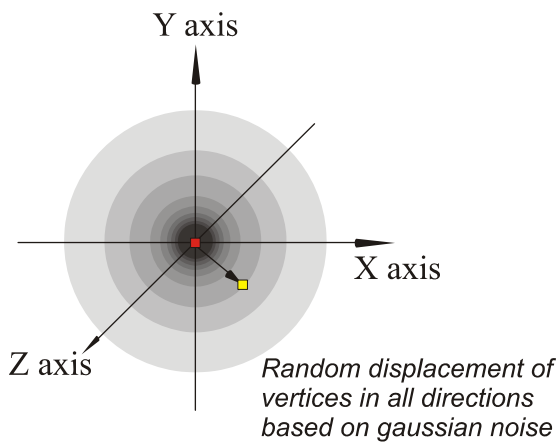

Figure 13 - Randomization Modes
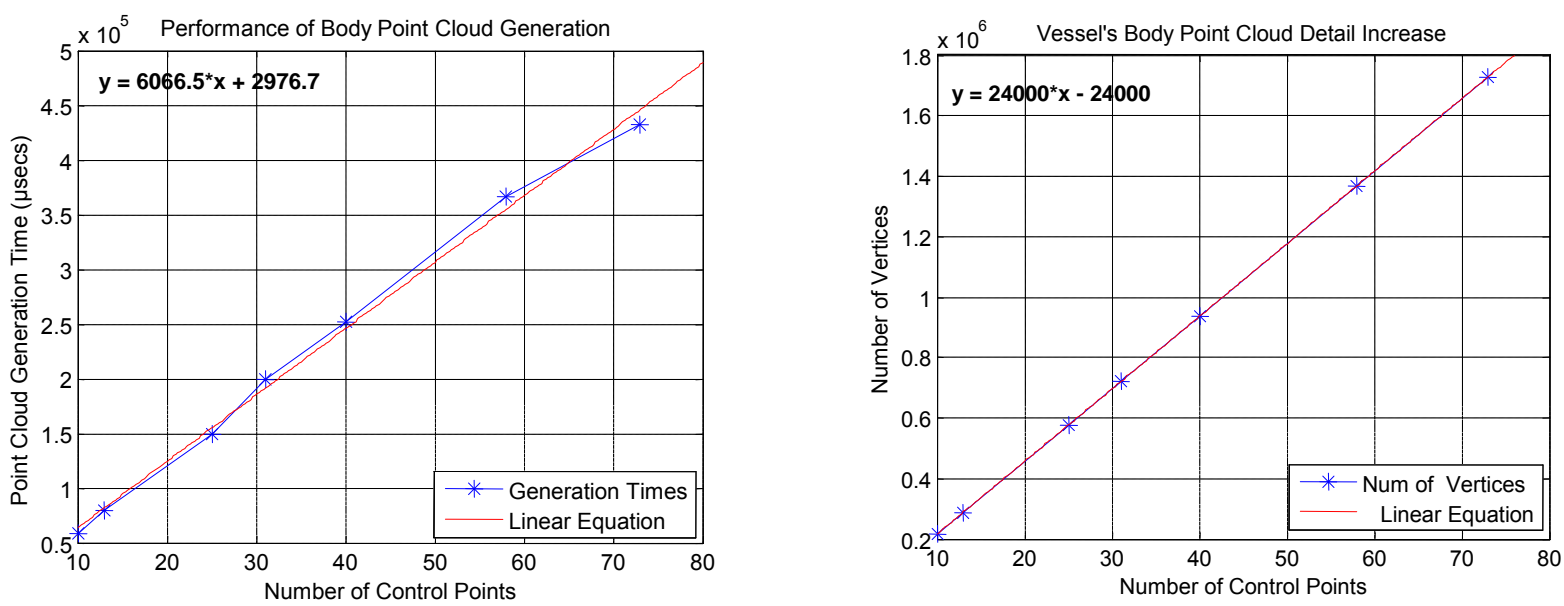

Figure 14 - Linear behaviour of revolve algorithm 

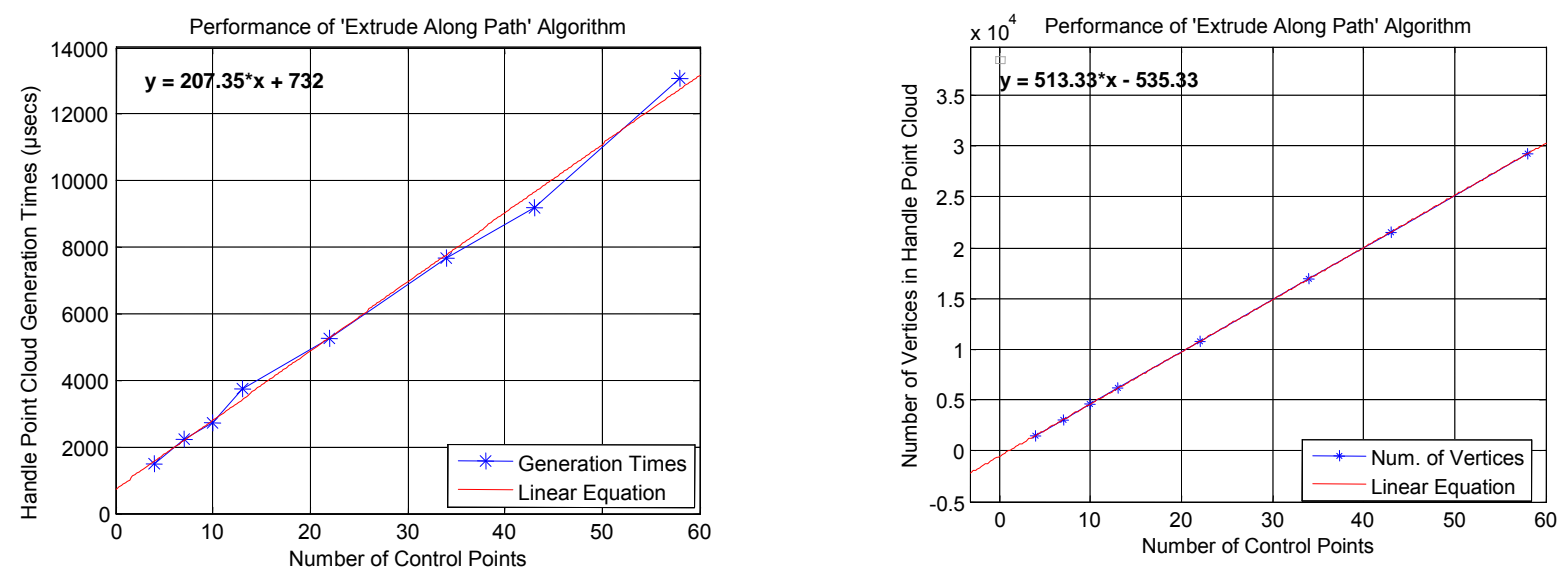

Figure 15 - Linear performance of extrude along path algorithm Performance of the Triangulation Algorithm

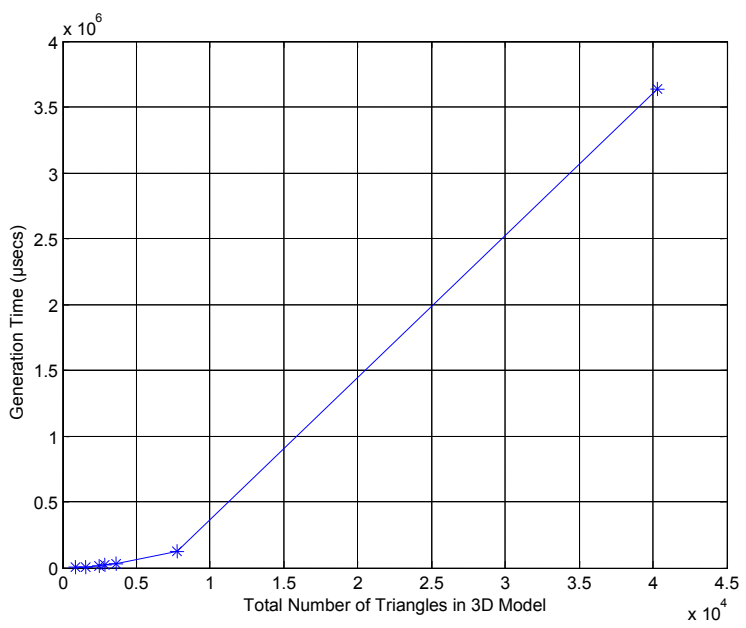

Figure 16 - The triangulation algorithm performance is described by a quadratic curve. 


\section{Tables}

TABLE I - Selected Shapes

\begin{tabular}{|c|c|c|}
\hline Shape Type & Shape Name & References \\
\hline & Alabastron & {$[23][24]$} \\
\hline & Amphora & [48][49] \\
\hline & Exaleiptron/plemochoe & [27] \\
\hline & Kalathos & {$[29][30]$} \\
\hline & Lebes/Dinos & [25] \\
\hline & Phiale & [26] \\
\hline & Psykter & [28] \\
\hline
\end{tabular}

TABLE II - ThreeDVDS - A 3D Vessel MPEG-7 Compliant Description Schema

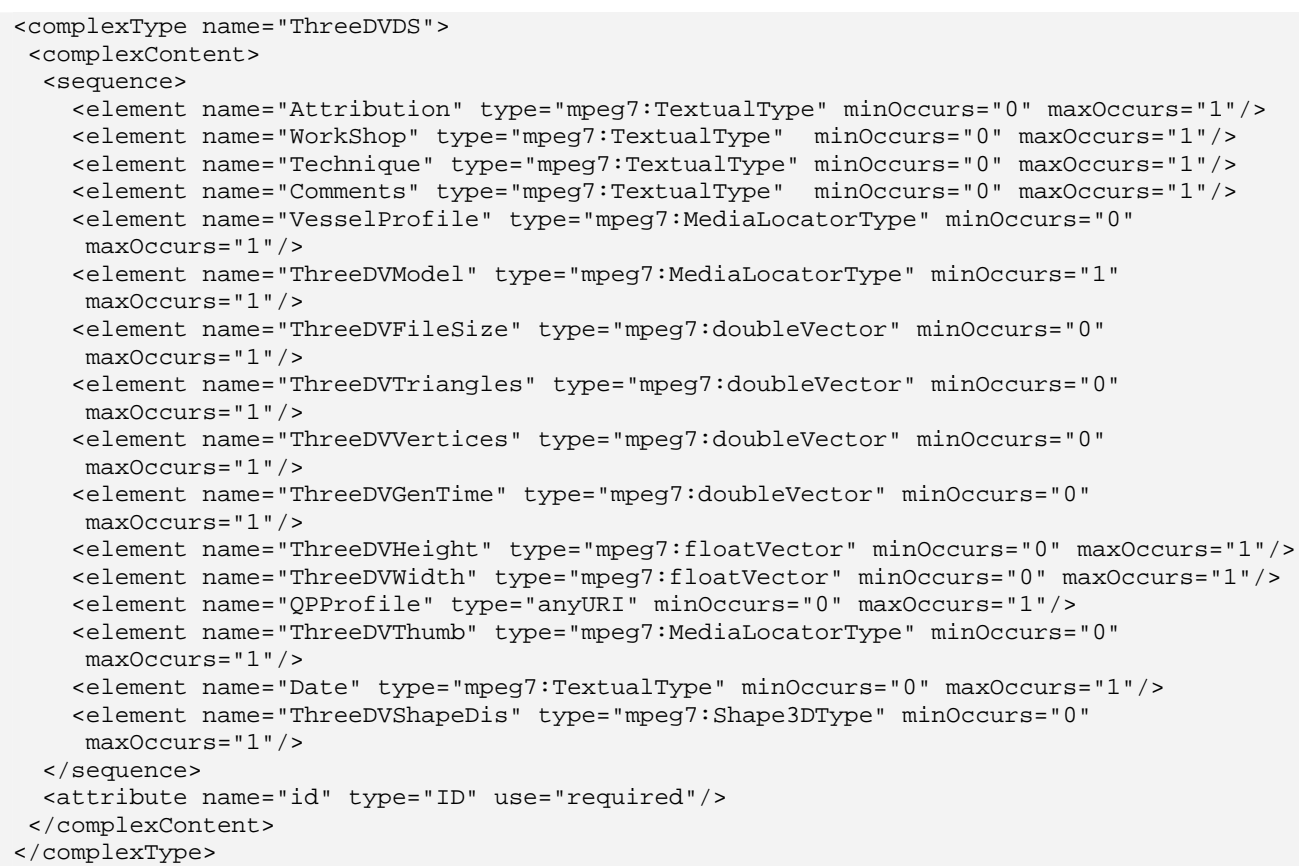

TABLE III - Description of Metadata in 3DVDS

\begin{tabular}{|l|l|}
\hline \multicolumn{1}{|c|}{ Element Name } & Description \\
\hline Attribution & Artist or Workshop to which the vase is attributed to \\
\hline WorkShop & Name of the artist's or city's Workshop to which the vase is attributed \\
\hline Technique & Type of painting technique \\
\hline Comments & Comments in textual format \\
\hline VesselProfile & The image that contains the 2D profile of the vessel \\
\hline ThreeDVModel & The path to the 3D model of the vessel \\
\hline ThreeDVFileSize & The file size of the 3D model \\
\hline ThreeDVTriangles & The number of triangles of the 3D model \\
\hline
\end{tabular}




\begin{tabular}{|l|l|}
\hline ThreeDVVertices & The number of vertices of the 3D model \\
\hline ThreeDVGenTime & $\begin{array}{l}\text { The time consumed for the generation of the 3D model (only applicable } \\
\text { to qp) }\end{array}$ \\
\hline ThreeDVHeight & The height of the 3D model \\
\hline ThreeDVWidth & The width of the 3D model \\
\hline ThreeDVThumb & The path to the thumbnail image of the 3D model \\
\hline QPProfile & The path to the profile file of the 3D model made with qp \\
\hline Date & The date the model was generated \\
\hline ThreeDVShapeDis & The 3D Shape Spectrum Histogram \\
\hline
\end{tabular}

TABLE IV - Random Vessel Generation Performance Analysis

\begin{tabular}{|c|c|c|c|c|}
\hline Shape Type & $\begin{array}{c}\text { Num. of } \\
\text { control } \\
\text { points }\end{array}$ & $\begin{array}{c}\text { Average } \\
\text { Generation Time } \\
\text { (seconds) }\end{array}$ & $\begin{array}{c}\text { Total 3D Data } \\
\text { size (bytes) }\end{array}$ & $\begin{array}{c}\text { Total } \\
\text { Metadata } \\
\text { size (bytes) }\end{array}$ \\
\hline 28 & 14.75 & $5,199,631$ & 63,511 \\
\hline Kabastron & 19.78 & $10,184,572$ & 62,548 \\
\hline Kalathos & $\begin{array}{c}20 \\
\text { (Body:16, }\end{array}$ & 17.65 & $6,823,123$ & 61,732 \\
\hline & 13 & 1.528 & $3,293,849$ & 62,299 \\
\hline Phiale & 7 & 1.564 & $1,445,481$ & 61,355 \\
\hline
\end{tabular}

